RUNNING HEAD: Forced Choice Measures with an Answer Key

\title{
Forced Choice Measures with an Answer Key: A Predictive Modelling Approach for Detecting Faking Behavior
}

10-08-2021

Angus W. Hughes ${ }^{1}$, Patrick D. Dunlop ${ }^{1,2}$, Djurre Holtrop ${ }^{3}$, Serena Wee ${ }^{1}$

${ }^{1}$ School of Psychological Science, University of Western Australia

${ }^{2}$ Future of Work Institute, Faculty of Business and Law, Curtin University

${ }^{3}$ Department of Social Psychology, Tilburg School of Social and Behavioral

Sciences, Tilburg University

This manuscript has been submitted for publication and is currently under review.

Correspondence about this paper can be addressed to:

Angus Hughes, School of Psychological Science, University of Western Australia, 35 Stirling Highway, Crawley, Western Australia 6009. ORCID: 0000-0002-0428-4085 Email: angus.hughes@ research.uwa.edu.au.

\section{Acknowledgements}

The authors would like to thank Eunike Wetzel for kindly providing the participant instructions in German for Study 2, as well as Susanne Frick and Anna Brown for making the data available. The first author was supported by a Jean Rogerson Postgraduate Scholarship, and an Australian Government Research Training Program Scholarship. 
FORCED CHOICE MEASURES WITH AN ANSWER KEY

\begin{abstract}
Forced choice (FC) personality measures are increasingly popular in research and applied contexts. To date however, no method for detecting faking behavior on this format has been both proposed and empirically tested. We introduce a new methodology for faking detection on FC measures, based on the assumption that individuals engaging in faking try to approximate the ideal response on each block of items. Individuals' responses are scored relative to the ideal using a model for rank-order data not previously applied to FC measures (Generalized Mallows Model). Scores are then used as predictors of faking in a regularized logistic regression. In Study 1, we test our approach using cross-validation, and contrast generic and job-specific ideal responses. Study 2 replicates our methodology on two measures matched and mismatched on item desirability. We achieved between $80-92 \%$ balanced accuracy in detecting instructed faking, and predicted probabilities of faking correlated with self-reported faking behavior. We discuss how this approach, driven by trying to capture the faking process, differs methodologically and theoretically to existing faking detection paradigms, and measure and context-specific factors impacting accuracy.
\end{abstract}

Keywords: Applicant faking, personnel selection, forced choice, personality, socially desirable responding. 
Despite decades of evidence that personality traits are predictive of important work outcomes, the use of personality measurement has long been viewed with skepticism amongst practitioners involved in personnel selection (Morgeson et al., 2007; Rees \& Metcalfe, 2003; Sackett \& Walmsley, 2014). A common concern is that a motivated individual intent on appearing competitive to a hiring committee may modify their honest responses in an attempt to better fit those expected of a highly desirable candidate (Ziegler et al., 2012). Perhaps 30$50 \%$ of job applicants are thought to engage in some degree of this so-called faking behavior (Griffith et al., 2007). If applicants are chosen on the basis of inaccurate idealized personality responses influenced by faking, employers may reasonably perceive risks of impaired personjob fit and poorer job performance (Wetzel et al., 2016). In efforts to improve personality measurement in applied contexts, considerable research attention has therefore been directed toward methods to both proactively prevent and retroactively detect faking (Kiefer \& Benit, 2016; Robie et al., 2006).

One of the most popular preventative strategies in recent years is the use of forcedchoice (FC) personality measures (Wetzel, Frick, \& Greiff, 2020). When completing a FC measure, participants must indicate which items within a block (typically comprising $2-4$ items) are most and least descriptive of themselves. If all of the items within a block provide an equally desirable impression (e.g., "I am diligent", "I learn quickly”), an applicant engaging in faking would likely find it difficult to identify the more desirable ordering of items (Zavala, 1965). Conversely, if the items in a block vary on their desirability (e.g., "I work hard", "I get frustrated easily"), a faker is likely to select the more desirable items as more like themselves, and the less desirable items as less like themselves. Meta-analytic evidence indicates the FC format does reduce the effects of faking at the trait-estimate level, as compared to Likert-type formats (Cao \& Drasgow, 2019). Nonetheless, faking on FC measures remains possible particularly where items have not been effectively matched for 
desirability. In practice, constructing blocks of equally desirable items may be challenging, as the desirability of an item likely depend upon the requirements of the job, and few commonly-used FC personality measures appear to use any form of context-specific item matching (Converse et al., 2010; Hughes et al., 2021b).

Rather than trying to proactively prevent faking, a separate line of research has attempted to retroactively detect faking based on participants' differential patterns of responding (Burns \& Christiansen, 2011). One promising approach introduced by Kuncel and Borneman (2007) involves evaluating differential patterns of responding under honest and job-applicant conditions. These authors first identified response patterns on 11 personality items indicative of faking (e.g., unexpectedly high/low endorsement of an item relative to what is typically observed under honest responding). An experimental sample were then instructed to respond either honestly or to fake good for an unspecified desirable job. Based on participants' use of these faking-indicative response patterns, the authors achieved roughly $70-82 \%$ accuracy in classifying participants instructed responding (honest, faking). Building on this, Calanna et al. (2020) recently illustrated how machine-learning algorithms can be used to analyze item-level response patterns and classify participants as either honest or faking. Their approach yielded predictive accuracies of around $74-76 \%$ in classifying individuals as faking good for either a fire fighter or teacher role. In sum, these studies have shown substantial potential for detecting faking by using an empirical index capturing distinct kinds of responding to personality items expected under faking behavior. Both studies, however, sought to detect faking exclusively on traditional Likert-type personality measures.

Despite the increasing popularity of the FC format, to date no study has experimentally tested the possibility of detecting faking on these types of measures. The key contribution of this study is to introduce a new predictive modelling approach to do so, using an empirical index based on responses expected under faking behavior, akin to the methods 
of Kuncel \& Borneman (2007) and Calanna et al. (2020). We propose that an applicant engaging in faking behavior will consistently try to provide a response resembling the ideal ranking on each block to make a better impression. Thus, for each block within a FC personality measure, our method involves (1) identifying the perceived 'ideal' answer to provide in a job applicant scenario, and (2) estimating the consensus about this ideal. Together, the ideal and consensus on each block form our own theoretically informed empirical index, or 'answer key'. We then evaluate the conditional probability of observing a particular response to a FC block, given the answer key, using a model for rank-order data (the Generalized Mallows Model, Fligner \& Verducci, 1986). The conditional probabilities of the rank-order responses on blocks across the FC measure are expected to be systematically higher for fakers, whose responses are liable to correspond to the answer key, than for those responding honestly. These probabilities thus form 'faking scores' for each participant's responses on each block, which are finally input into a logistic regression to classify a participant's responding overall as honest or faked. The following sections outline in more detail both the theoretical and methodological bases of our approach.

\section{Providing the Ideal Rank-Order as a Faking Strategy}

At its core, our approach is built upon the assumption that each FC block has an 'ideal' response, which participants who fake are trying to approximate. The ideal is defined as the rank-ordering of items that the average applicant would feel makes the most desirable impression to a hiring committee. The process of trying to identify the most desirable response on each personality item is consistent with how many individuals report their faking strategy in qualitative studies (König et al., 2012; Robie et al., 2007). Clearly individuals' subjective perceptions of what constitutes the ideal response may differ from the average ideal, however collecting individual-level ideal responses is impractical for the purposes of faking detection in applied settings. Our ideal instead draws on the 'wisdom of the crowd' to 
provide the most reasonable aggregated rank-ordered response, averaging across individual perceptions of desirability (M. D. Lee et al., 2014). Evidence does suggest that individuals' inconsistency with the aggregated ideal response to blocks on a FC measure tends to correspond to deviations from their honest responses under fake-good instructions (Hughes et al., 2021a). Note however that the ideal does not explicitly consider the specific personality traits being measured within each block and ultimately selected for, as the scoring of personality measures are almost always unknown to the respondent (König et al., 2017).

If we identify for each block of items the ideal rank-ordering, variability in the consensus amongst individuals regarding this rank-ordering needs to be accommodated. For example, the ideal rank-ordering of responses would probably seem obvious in blocks where items are extremely mismatched in terms of desirability, meaning most individuals engaging in faking behavior would provide the same answer (for example, see $\mathrm{Ng}$ et al., 2020, p. 10). On the other hand, in the case of a hypothetical perfectly matched block, all possible rankorderings should be perceived as equally ideal, meaning no consensus emerges. In principle, perfectly matched blocks should be more faking-resistant, however these may be difficult to craft in practice (although for guidance on matching, see Pavlov et al., 2021). Many commercial FC measures appear to be matched for desirability in a fixed, one-size-fits-all manner, rather than for their desirability to a specific job context; job-specific matching would likely necessitate intensive measure design and validation (Converse et al., 2010). Item blocks even on a very well-crafted FC measure are thus unlikely to be perfectly matched across all job contexts, resulting in varying degrees of consensus between individuals regarding the ideal rank-ordering (Hughes et al., 2021b).

\section{Evaluating Participants' Rank-Order Responses Relative to the Ideal}


Our approach rests on the assumed difference in responding processes between individuals faking and those responding honestly. We propose that the process by which individuals engage in faking is by either modifying or completely fabricating their own responses in a way that better resembles the perceived ideal response. In contrast, it seems unlikely that an individual's honest responding should be systematically influenced by the perceived ideal response for the purposes of impressing a hiring committee across every single block on the FC measure. Precisely because providing responses that correspond with the ideal answer in a job application scenario consistently across the entire FC measure is unrealistic for honest responses, yet plausible for faked responses, we base our approach on evaluating all of an individuals' responses relative to the ideal. We accomplish this by using the Generalized Mallows Model (GMM; Fligner \& Verducci, 1986), an established probability model for rank-ordered data. The GMM, in our case, models the probability of observing a rank-ordering, $\pi$, given the perceived ideal rank-ordering, $\pi_{0}$, and the level of consensus, $\theta_{j}$, regarding the $j$-th ranked item in $\pi_{0}$.

The GMM is based upon distances between rankings. For example, consider an ideal rank-ordering, $\pi_{0}$, on a hypothetical FC block with $m=3$ items (a 'triplet') consisting of " $I$ am organized" > "I am tidy" > "I like music", and a hypothetical participant's response, $\pi$, of "I am tidy" > "I like music" > "I am organized". The distance of this participant's response, $\pi$, relative to the item ranked in first place $(j=1)$ in the ideal rank-ordering is $d_{j=1}\left(\pi, \pi_{0}\right)=2$, because two items - 'music' and 'tidy' - were ranked above 'organization'. With the distance relative to first-ranked item in $\pi_{0}$ counted, now the distance from the second item is $d_{j=2}\left(\pi, \pi_{0}\right)=0$, because 'tidy' was ranked by the participant above 'music' consistently with the ideal, $\pi_{0}$. The distance function, $d_{j}$, adopted here is defined as the number of items in a given rank-order, $\pi$, ranked above the $j$-th ranked item in the ideal rankorder, $\pi_{0}$ (Mandhani \& Meila, 2009). 
The GMM then incorporates consensus regarding the ideal rank ordering, $\pi_{0}$, in defining the probability of each possible rank-ordering as:

$$
P\left(\pi \mid \pi_{0}, \theta\right)=\frac{\exp \left(-\sum_{j=1}^{m-1} \theta_{j} d_{j}\left(\pi, \pi_{0}\right)\right)}{\psi(\theta)}
$$

Where the $\theta_{j}$ parameters relate to the distance function to control the strength of consensus that the $j^{\text {th }}$ ranked item in the ideal rank-ordering $\pi_{0}$ should be ranked above the other remaining items. Then, $\theta=\left(\theta_{1}, \ldots, \theta_{m-1}\right)$ and $\psi(\theta)$ is a normalizing constant. For a set of $m$ items, the GMM thus defines a probability distribution over all $m$ ! possible rankorderings in terms of $m-1$ distances from $\pi_{0}$. To illustrate, on a hypothetical three-item block ( $m=3$ ), the probability values of all $m$ ! possible rankings at differing levels of $\theta$ are presented in Table 1. Increases in $\theta$ correspond to stronger agreement as to the 'proper' position of the $j$-th ranked item in $\pi_{0}$, such that the probability of observing a rank-ordering of items differing from this becomes minimal (Irurozki et al., 2016). Large $\theta_{j}$ values are likely to reflect mismatched blocks, whereas well-matched blocks should have smaller $\theta_{j}$ values.

Our approach involves evaluating the GMM-derived conditional probability of observing a participant's rank-ordered response to each block across the FC measure. We essentially treat these conditional probabilities as 'faking scores' for each block. If individuals do fake by consistently approximating the ideal response, on average we would expect these faking scores to be higher across the measure as compared to individuals responding honestly. Estimating the $\pi_{0}$ and $\theta$ parameters for a given block to score a response against the GMM requires data from an independent sample tasked with ranking the desirability of the items. Such a sample could be instructed to either rank the desirability of the items in any given job applicant (mirroring the generic, one-size-fits-all approach to 
matching discussed above), or for the specific job. We presuppose that as the desirability of each item may be contextual to the role being applied for (Dunlop et al., 2012; Pauls \& Crost, 2005), a job-specific answer-key may better correspond to the responses provided by individuals engaging in faking.

\section{Predicting Faking Good: Maximizing Out-of-Sample Accuracy}

An individual's faking scores from each block can be combined to classify their responding across the measure as either honest or faked. A simple sum or average of the faking scores across the blocks could fit this purpose but would take for granted that all blocks are equally diagnostic of faking behavior. Although the magnitude of GMM-derived faking scores incorporate consensus within each FC block regarding the ideal response, when looking across the FC measure as whole, certain blocks may disproportionately elicit responses closer to the ideal under faking conditions relative to honest conditions. We therefore use a logistic regression to assign weights to the faking scores on each block. To fit this model, we use data from a faking study wherein participants are explicitly instructed to either respond honestly or fake good for a hypothetical job application. The logistic regression model is 'trained' to predict the probability that an individual was assigned to the faking-good instructions, using their faking scores on each block as predictors.

Nonetheless, simply training a model with numerous predictors on a given dataset risks overfitting, wherein idiosyncratic characteristics of the observed data generalize poorly when trying to predict the faking outcome on a new sample (Kuhn et al., 2013, pp. 61 - 64). We therefore adopt two strategies to handle overfitting. First, we employ regularization, which shrinks the magnitude of (i.e., penalizes) predictors to improve model generalizability (James et al., 2013, pp. 214 - 226). Second, we use cross-validation methods to both enhance and test out-of-sample accuracy. Here, we randomly subset a large proportion of the faking 
study data (training data) for model fitting purposes, while holding out the data left over to evaluate our model (testing data). Using just the training data, we conduct $k$-fold repeated cross-validation to calibrate the model parameters, iteratively searching for the logistic regression coefficients and penalties fitted to random subsets of this data that maximize outof-sample predictive accuracy. Finally, we quantify the out-of-sample classification accuracy by predicting the faking good outcome in the testing data using the optimal model fit. Below, we illustrate the utility of both strategies in two separate studies.

\section{Study 1}

To evaluate the effectiveness of our approach, we formed three broad research questions. First, can GMM-derived faking scores using the answer key for a particular job effectively predict whether a person completing a FC measure was instructed to respond honestly, or instructed to fake-good for a job application? If so, researchers and practitioners may be able identify respondents engaging in faking for a job on FC measures. Second, does a generic answer key, containing ideal responses applicable for any given job (as opposed to a specific job), yield similar predictive accuracy as a job-specific answer key? While previous research indicates that the ideal answer may depend upon the job, if a generic answer key is approximately as effective at identifying fakers this would mitigate the potentially large investment in identifying ideal responses for specific jobs and training separate GMM/logistic models for each. Third, do our predictions of faking behavior correspond to the extent of self-reported faking behavior amongst those instructed to fake good for a particular job? If so, we may be able to substantiate our behavioral assumption underlying the method, namely that those predicted as faking (based on higher GMM faking scores across FC blocks) also reported engaging intentionally in more faking behavior.

\section{Method}


All data collection was conducted following approval by the Human Research Ethics Office of the University of Western Australia. All study materials are available via the Open Science Framework (OSF; https://osf.io/3f97g/).

\section{Measures}

Forced Choice Personality Measure. The FC measure comprised 48 three-item blocks (hereafter 'triplets'; 144 items total), with items measuring separate Big 5 traits, containing both positively and negatively keyed statements. The items forming the triplets were not all matched on desirability, but rather systematically varied in how well-matched they were on generic job-related desirability (i.e., for any given job). More details on the development of this measure are provided in Hughes et al. (2021). Triplets were presented in a randomized order across all data collections.

Self-Reported Faking. After completing the personality measure under instructed faking, participants were asked the extent to which they manipulated their responses to appear better for the job on a 7-point Likert scale, ranging from "not at all - my answers were completely honest" to "completely - none of my answers were honest" $(M=4.5, S D=1.61)$.

\section{Participants and Procedures}

Three separate samples from the United States were recruited via Amazon Mechanical Turk (MTurk). These were the: A) Ideal ranking sample; B) Honest ranking sample; and C) Faking sample. The data from sample A was previously collected and reported in Hughes et al. (2021), and samples A - C in Hughes et al. (2021a) for separate research studies. Details of each sample (in order of their collection) and procedures are described below.

Ideal ranking sample. Participants in this sample were randomly allocated into one of three job conditions and tasked with ranking the desirability of the items comprising each 
triplet in a lawyer applicant $(N=102)$, kindergarten teacher applicant $(N=107)$, or a generic applicant for any given job $(N=109)$. Participants in the lawyer and teacher conditions were provided a detailed description of their assigned job, along with key responsibilities and hiring criteria, based on materials from $\mathrm{O}^{*} \mathrm{NET}$. More details on this sample can be found in Hughes et al. (2021).

Honest sample. Participants $(N=322)$ in this sample completed the FC measure under honest responding instructions. Participants were told to "answer as yourself, in terms of how you normally think, feel, and behave" and that "there are no right or wrong answers". Individuals were removed for failing two separate attention checks, voluntarily flagging their responses as untrustworthy, engaging in suspected careless responding (based on long-string patterns, psychometric synonyms and antonyms, implausibly fast completion times; Curran, 2016), or appearing in the faking sample (below). Thus, a total of 215 participants were retained.

Faking sample. Participants $(N=301)$ were recruited for a two-wave faking study. Participants were randomly allocated in wave 1 to respond to the FC measure either honestly or faking good for a job application (details below). In wave 2, participants were then allocated to the opposite condition, such that all participants responded under both sets of instructions in a counterbalanced order. When tasked with responding as a job applicant faking good, participants were further allocated to respond either in relation to an application for a lawyer role $(N=101)$ or a kindergarten teacher role $(N=116)$.

In the honest condition, participants received the same instructions as the honest sample described above. When faking good for a job application, participants received the same detailed description of the job, responsibilities, and hiring criteria as the ideal ranking sample described above. Participants were then explicitly asked to respond, "in the way you 
think would maximize your chances of being selected for the role." At the end of each wave, participants were asked to recall their responding instructions for that wave as an attention check. Upon the completion of the job applicant condition, participants who successfully recalled their instructions then completed the self-reported faking measure.

After completing wave 1, we removed 47 suspected careless responders on similar criteria as described above. Of the 254 participants reinvited, 223 participants completed wave 2 approximately one week later. Removing another 6 suspected careless responders resulted in a final sample size of $N=217$ completing both waves $\left(\mathrm{M}_{\text {age }}=42.8, \mathrm{SD}=12.6\right.$, Range 18 - 77; 119 males, 97 females, 1 non-identified).

\section{Analysis Plan}

Generalized Mallows Model Estimates. Using the data from the ideal ranking sample, we fit a GMM to each triplet comprising our measure. This meant separately estimating both the ideal rank-ordering, $\pi_{0}$, and two consensus parameters, $\theta_{j}$, for the ideal ranking sample's lawyer, teacher, and generic job applicant responses on each triplet. These estimates formed our three different 'answer keys', one per job applicant condition. The ideal rank-ordering $\pi_{0}$ was determined using the Kemeny-Young method, which identifies the rank-ordering minimizing the overall rank distance between the ideal and all observed rankings. This method was implemented using a branch-and-bound algorithm from the "ConsRank" package for R (D’Ambrosio, 2019). The GMM consensus parameters, $\theta_{j}$, for the first two items in each triplet by job condition were determined by maximum likelihood using the “PerMallows” R package (Irurozki et al., 2016).

Average Kendall's Tau. The sum of the rank distances used in fitting the GMM yields the overall Kendall's Tau distance between two rank-orders, $\sum_{j=1}^{m-1} d_{j}\left(\pi, \pi_{0}\right)=\tau_{d}$. This $\tau_{d}$ counts the total number of pairwise disagreements between two rankings on the 
ordering of each pair of items, and for a triplet ranges from 0 to 3 . A $\tau_{d}$ can in turn be normalized to find the Kendall's $\tau$ correlation (Kendall \& Gibbons, 1990, pp. 6 - 7) between two rankings:

$$
\tau=1-2\left(\frac{1}{\tau_{D \max }}\right) \tau_{D}
$$

Where $\tau_{D \max }=m(m-1) / 2$. As such, to quantify the overall consensus on each triplet regarding the ideal ranking ordering $\pi_{0}$ as a descriptive statistic on a more familiar scale, we also estimated the average Kendall's $\tau$ correlation between $\pi_{0}$ and each respondents' perceived ideal rank-orderings. An average Kendall's $\tau$ of 0 implies no consensus amongst the sample regarding the ideal, whereas $\tau=1$ implies complete consensus.

Faking Scores on Forced-Choice Responses. To score our FC personality measure data for faking behavior in both the honest and faking samples, we computed the conditional probabilities of each observed rank-order against the relevant answer key again using "PerMallows" (Irurozki et al., 2016). This faking score data from both samples was then split three ways based on the answer key, forming a lawyer dataset $(N=331$ responding honestly, $N=101$ faking $)$, a teacher dataset $(N=316$ honest, $N=116$ faking $)$, and generic-job dataset ( $N=215$ honest, $N=217$ faking). For example, all those included in the lawyer dataset had their FC responses scored relative to the ideal answers in a lawyer applicant. If the assigned job condition of individuals in the faking sample corresponded to the answer key of the dataset, only their scored faking responses were included in the data, otherwise their scored honest responses were used. In the generic dataset, only the faking responses of those in the faking sample were included, all scored relative to the same generic answer key. All responses from the honest sample were included in each dataset, again scored relative to the 
respective answer key. Each dataset thus comprised responses from $N=432$ independent participants.

Predicting Faking Responses. We used regularized logistic regression models to determine the optimal weightings for each triplet that best predict the probability an individual was faking. Participants' faking scores on each of the 48 triplets were used as predictors of the participant's instructed responding (honest, faking) in three such models, one for each dataset described above. We employed elastic net logistic regression, which uses a mix of two penalty terms (the lasso, $L_{1}$ and ridge, $L_{2}$ ) to effectively shrink the coefficients of less predictive triplets in classifying a response as either 'faking' or 'honest' (Zou \& Hastie, 2005). We used the "glmnet" R package (Friedman et al., 2010) to fit elastic net regression models.

We chose to train our models on approximately $2 / 3^{\text {rds }}$ of the sample for each dataset (lawyer, teacher, generic). This training data was then randomly split into 10 folds, with cross-validation repeated 5 times at different penalty terms to determine the model parameters that maximized predictive accuracy. The cross-validation method is visually depicted in Figure 1. As the lawyer and teacher datasets were imbalanced on responses (approximately 3:1 in favor of honest responses), which can negatively impact model performance, during training we resampled faking responses with replacement (i.e. upsampling, Kuhn et al., 2013, p. 427) to equate the number of observations per fakinginstruction outcome (honest, faking). We then applied our models to make predictions for the remaining $1 / 3^{\text {rd }}$ of the sample (i.e., the testing data; 143 observations). The threshold for classification of faking in this testing data was set at a predicted probability of $\geq .50$. For example, an individual with a predicted probability of .51 would be classified as 'faking'. Next, for each model's predicted classification on the testing data, we tabulated the faking respondents that were correctly classified (true positives, TP), honest respondents incorrectly 
classified as faked (false positives, FP), honest respondents correctly classified (true negatives, TN) and faking respondents incorrectly classified (false negatives, FN). The “caret” package was used for all model cross-validation (Kuhn, 2020).

To calculate out-of-sample predictive performance, we calculated four metrics. Sensitivity, defined as $T P /(T P+F N)$, estimated the model's ability to correctly classify fakers. Specificity, defined as $T N /(T N+F P)$, estimated the correct classification rate of honest respondents. Balanced accuracy, defined as (Sensitivity + Specificity)/2, was used for overall model predictive performance while accounting for imbalanced proportions of honest to faking respondents in the test data. Finally, we plotted the receiver operating characteristic (ROC) curve, which shows trades-offs between sensitivity and specificity across a range of possible classification thresholds and calculated the area under the (ROC) curve (AUC) to index the model's ability to discriminate between honest and faking responses. All four metrics (sensitivity, specificity, balanced accuracy, and AUC) are maximized at 1.0. ROC and AUC metrics were calculated using the "pROC" package (Robin et al., 2011).

\section{Results}

The GMM $\theta_{j}$ parameter estimates and average Kendall's $\tau$ for each FC triplet and job condition are presented in Figure 2. As shown, there was a high degree of variability in the extent of consensus on the ideal response. Larger $\theta_{j}$ values indicated higher consensus amongst the ideal ranking sample regarding the desirability of the first and/or second ranked items in the ideal response, $\pi_{0}$. The average Kendall's $\tau$ correlation between $\pi_{0}$ and the ideal ranking sample data for each block captured the overall consensus on each triplet, and ranged from .02 to .87 , reflecting exceptionally well-matched and poorly matched triplets respectively. Next, we calculated the mean GMM-derived faking scores within individuals instructed to fake and those instructed to respond honestly. These mean GMM faking scores 
across the FC measure are presented in Figure 3. Observing the differences in mean faking scores between these instructions yielded a Cohen's $d=2.65,95 \%$ CI [2.27, 3.03], in the lawyer condition, $d=2.47,95 \%$ CI $[2.15,2.79]$, in the teacher condition, and $d=1.54,95 \%$ CI $[1.33,1.76]$, in the generic job applicant condition. In other words, the average faking scores across all triplets were typically much higher amongst individuals instructed to fake.

Results of all elastic net logistic regression models in terms of both within-sample (training) and out-of-sample (testing) predictive classification performance are presented in Table 2. Our models of instructed responding (honest, faking) showed strong predictive performance using the GMM-derived faking scores. Models using job-specific faking scores achieved an average within training sample AUC of around $.96-.97$ for the lawyer condition and teacher conditions across 5 repeats of $k=10$-fold cross-validation training phases. The balanced accuracy in the training data of each of these models was around $.89-90$, with similar results in the testing data suggesting good out-of-sample predictive performance. The generic model was slightly less accurate than the models using job-specific faking scores, attaining a balanced accuracy of .85 in both the training and testing data, and a within training sample AUC of .94. Full ROC curves across a range of sensitivities and specificities are presented in Figure 4.

Finally, to test whether the model corresponded to individuals' subjective faking behavior, we calculated model-based predicted probabilities for the responses of individuals instructed to fake. These were found to correlate with self-reported faking using probabilities from the lawyer model, $r(98)=.37,95 \%$ CI $[.19, .53], \mathrm{p}<.001$, the kindergarten teacher model, $r(112)=.43,95 \% \mathrm{CI}[.27, .58], \mathrm{p}<.001$, and generic model, $r(212)=.31,95 \% \mathrm{CI}$ $[.18, .43], \mathrm{p}<.001$. The relationship between predicted faking and self-reported faking across both datasets is visualized in Figure 5. As shown, at higher levels of self-reported faking, all 
models increasingly predicted the probability of those instructed to fake as faking, although this relationship was weaker when using the generic model.

\section{Study 2}

The results of Study 1 were highly encouraging, exceeding the accuracy of previously introduced Likert-based approaches (Calanna et al., 2020; Kuncel \& Borneman, 2007). However, a key concern around the generalizability of our remarkably strong predictive performance must be noted. The blocks comprising the FC measure used in Study 1 were explicitly designed to vary in terms of how well-matched the desirability of the items were. While some blocks were exceptionally well-matched on how desirable the items would appear in a job applicant, many blocks had an obvious 'best' answer where the vast majority of people would agree on the ideal ordering of the items (Hughes et al., 2021b). Accordingly, one potential criticism is that the FC measure used in Study 1 was unrepresentative of those that would typically be used for high-stakes assessment contexts. We also had reason to suspect this design aspect of the FC measure may have contributed to our high degree of classification accuracy. Poorly-matched blocks could plausibly help discriminate between instructed faking and honesty, relative to blocks where all items are perceived as more equal in terms of their desirability. Applying our method to a FC measure where all blocks were purposely designed to be matched on desirability may therefore yield less accuracy in classifying fakers.

To address the concerns above, we next sought to replicate our approach in an independent dataset from a previously conducted study by Wetzel, Frick, \& Brown (2020). This study used an extensively validated FC personality measure comprising 20 triplets, purposely matched on social desirability (Wetzel \& Frick, 2020). The authors then modified seven of their twenty triplets to be systematically mismatched on desirability, forming a 
separate measure to test the influences of item matching on faking. We recruited a new sample to generate perceived ideal rankings on each triplet for both measures. As our models employing job-specific answer keys appeared to provide the best results in Study 1, the ideal rank-orderings we collected were for the purposes of impressing a selection panel assessing prospective Masters Students in Psychology (the role used by Wetzel, Frick, \& Brown, 2020). Then, mirroring our method as detailed in Study 1, we tested whether our approach could identify those instructed to fake for a different role, amongst a separate sample, on two FC measures specifically matched and mis-matched on item desirability.

\section{Method}

All data collection was conducted following approval by the Human Research Ethics Office of the University of Western Australia. All study materials are available via the Open Science Framework (OSF; https://osf.io/3f97g/).

\section{Measures}

Big Five Triplets (BFT) - Matched. This German-language FC measure consists of 20 triplets, containing positively and negatively keyed statements, and measuring separate Big 5 traits within each triplet. To develop this measure, the authors recruited 33 German psychology students to rate the social desirability of each item individually on a 5-point Likert scale (ranging from 'socially undesirable' to 'socially desirable'). Items were then matched on their median desirability rating, with medians falling between two Likert-scale points (e.g., 4.5) resolved by setting the ratings as the more frequent category. Extensive details on the development and validation of this measure are presented in Wetzel \& Frick (2020).

Big Five Triplets (BFT) - Mixed. The second FC measure was identical to the BFTmatched measure described above, with the exception of the first seven triplets. For the 
purposes of the study conducted by Wetzel, Frick, \& Brown (2020), one socially undesirable or neutral item in each of these triplets was replaced with a highly desirable item to introduce systematic mismatching on item desirability. The item replacements are available via the OSF at https://osf.io/5s43z/. Because the last 13 triplets comprising the measure remained matched on desirability, it was referred in the original publication as the BFT-mixed.

\section{Participants and Procedures}

Two separate samples were used for this second study. The first sample was from Wetzel, Frick, \& Brown (2020), who recruited participants via two German universities and Respondi, a German panel data company. The second sample was recruited via MTurk and Prolific (www.prolific.co) from eligible participants in Germany, Austria, and Switzerland. Details of each sample (in order of their collection) and procedures are described below.

Faking study sample. Participants, aged between 18 - 30 and speaking German as their first language, were eligible for participation; exclusion criteria, including careless responding indices, are described in detail in Wetzel \& Frick (2020). The original dataset, in paired comparison format, was obtained via the OSF at https://osf.io/q9uyp/. Participants were randomly assigned to complete either the BFT-matched $(N=593)$ or the BFT-mixed $(N$ $=652)$ twice in one testing session: first under honest responding conditions, and then under instructed faking conditions for an application to a Master's Program in Psychology. Under the fake-good condition, participants were provided a description of the selection criteria and instructed to answer in a manner that made them appear more open, conscientious, extraverted, agreeable, and emotionally stable to increase their chances of acceptance into the program (full instructions are reproduced in Wetzel, Frick, \& Brown, 2020, p. 12). A total of 34 individuals failed to respond to $\geq 40 \%$ of the BFT blocks under honest and/or faking instructions and were removed from our analysis. As each participant completed their 
assigned version of the BFT twice under the two instruction sets, we randomly selected whether an individual's honest or faked responses would be used within the models to ensure independence of observations. The final sample size was therefore $N=577$ for the BFTmatched and $N=638$ for the BFT-mixed.

Ideal Ranking Sample. Participants were eligible for participation in our ideal ranking sample if they spoke German as a first language and resided in one of the three selected German-speaking countries, with proficiency in German screened at the beginning of the survey. After eliminating one participant for suspected careless responding, the final sample comprised $N=109$ individuals $\left(\mathrm{M}_{\mathrm{age}}=29.9\right.$ years, $\mathrm{SD}=7.95$, Range $=18-60 ; 26$ females). Participants were first instructed to rank how desirable the statements comprising each block on both the BFT-matched and mixed (27 triplets total) would look in an applicant for a Master's degree in Psychology, without reference to their own personality. They were then provided exactly the same selection criteria and instructions for faking as that of Wetzel, Frick, \& Brown (2020), and completed each block in a randomized order. Each triplet was ranked by on average $N=99$ participants.

\section{Analysis Plan}

Generalized Mallows Model Estimates and Average Kendall's Tau. Mirroring the methods of Study 1, we used data from the ideal ranking sample to estimate for each BFT mixed/matched block both the ideal rank-ordering, $\pi_{0}$, and two $\theta_{j}$ consensus GMM parameters. We also calculated the average Kendall's $\tau$ correlation between the ideal ranking sample responses and $\pi_{0}$ in an identical manner to Study 1.

Faking Scores on Forced-Choice Responses. To score the faking study data, we computed for each individual's honest and faked responses the probability of the observed rank-order, given the GMM parameters. As a small number of responses were missing 
$(113 / 48,600=0.23 \%)$, which cannot be handled by 'glmnet', we opted to use multiple imputation by chained equations (MICE, Azur et al., 2011) to generate 5 imputed datasets, and run all subsequent analyses on each.

Predicting Faking Responses. Participants' faking scores on each of the 20 BFTmatched/mixed triplets were used as predictors of the participants' instructed responding (honest, faking) in two elastic net logistic regression models, one for each FC measure. We then held out approximately $1 / 3^{\text {rd }}$ of the data to test our model predictions $(192 / 577$ observations for the BFT-matched, 212/638 for the BFT-mixed), and trained our model on the remaining $2 / 3^{\text {rds }}$ of the data, again employing a $k=10$ fold cross-validation approach, repeated 5 times. Both the mean within-sample and out-of-sample predictive performance were assessed using balanced accuracy, sensitivity, specificity, and the AUC metrics, averaged over the 5 imputed datasets.

\section{Results}

The GMM $\theta_{j}$ parameter estimates and the average Kendall's $\tau$ for each BFT measure given the ideal rank-ordering, $\pi_{0}$, on the triplet, are presented in Figure 6. In general, for triplets $1-7$ the average Kendall's $\tau$ noticeably increased in the BFT-mixed measure, indicating higher consensus amongst the ideal ranking sample on these systematically mismatched blocks. The highly desirable item introduced to each of these triplets (replacing the undesirable or neutral item in the BFT-matched measure) was typically ranked first in $\pi_{0}$, with the larger $\theta_{j=1}$ parameters indicating a high degree of consensus on this item's desirability. Nonetheless, some consensus emerged regarding the ideal response even in the BFT-matched measure, with around 8 triplets attaining an average Kendall's $\tau$ of $\geq .50$. The mean GMM faking scores for each individual across the FC measure are presented in Figure 7. The differences in mean faking scores between response instructions (honest, faking) 
yielded a Cohen's $d=1.45,95 \%$ CI $[1.26,1.63]$, on the BFT-matched measure, and $d=1.47$, 95\% CI [1.29, 1.64], on the BFT-mixed measure. The average faking scores across all triplets were therefore higher amongst individuals instructed to fake on both measures.

Results of both the BFT-matched and BFT-mixed models in terms of both withinsample (training) and out-of-sample (testing) predictive classification performance are shown in Table 3. All classification metrics were noticeably reduced compared to Study 1.

Nonetheless, the elastic net logistic regression model using the GMM-derived faking scores showed good performance in predicting faking, achieving a maximum within-sample AUC of 0.88 for the BFT-matched and 0.89 for the BFT-mixed during 5 repeats of the $k=10$-fold cross-validation training. The average balanced accuracy of each model was around $.81-.82$ during training, with similar results in the testing data. Full ROC curves across a range of sensitivities and specificities are presented in Figure 8. Overall, the predictive performance across a range of classification thresholds of the BFT-matched and BFT-mixed models did not appear to differ substantially. The lower sensitivity, relative to specificity, in both models implied that more participants instructed to fake tended to be misclassified as 'honest' (i.e., inflating the false negative rate). On the other hand, the higher specificity meant there were fewer false positives in wrongly flagging a participant responding honestly as 'faking'.

\section{General Discussion}

We developed a novel method of identifying faking on FC personality questionnaires, based on the rationale that people engage in faking behavior by attempting to provide an idealized response on each FC block. If the response to each block that most individuals would feel provides the best impression to a hiring committee (i.e., the ideal response) is identified, we can compare this ideal 'answer key' to an individual's responses across the FC measure to predict whether they are faking. We repurpose an established model for rank- 
ordered data, the GMM (Fligner \& Verducci, 1986), to evaluate the conditional probability that an individual generated their response in reference to the ideal rank-ordering on a given FC block, which incorporates variability in perceptions of the ideal response. Finally, we treat these conditional probabilities on each block as 'faking scores' and use elastic net logistic regression, trained on a faking study dataset, to assign regression weights to each block and generate a predicted probability of faking. Across two separate studies, we demonstrate that this approach can yield good to excellent out-of-sample classification accuracy (80-92\%), and that model predictions correlate with self-reported faking behavior.

In terms of predictive performance, our results compare favorably to previous attempts to identify fakers, which have exclusively used Likert-type questionnaires in instructed honest and faking good faking study designs. For example, methods using Likert item-covariance based indices achieved around 63.5\% accuracy (Christiansen et al., 2017), hybrid Rasch-latent class modelling up to $65.7 \%$ accuracy (Holden \& Book, 2009), combined structural equation modelling and person-fit measure approaches roughly $80 \%$ accuracy (Ferrando \& Anguiano-Carrasco, 2013), and item response process tree methods around $71.6 \%{ }^{1}$ balanced accuracy overall (with some subscales as high as $86 \%$, Sun et al., 2021). We note that none of these studies employed cross-validation, meaning these reported results may in fact have overestimated out-of-sample accuracy. Calanna et al. (2020) utilized a method conceptually closest to ours, achieving a cross-validated accuracy of $76 \%$ using response patterns indicative of faking on Likert-type scales as predictors in a logistic regression. Our results for both studies appeared to meet and exceed both this and indeed the vast majority of previously published methods of faking detection in terms of accuracy, and to our knowledge empirically demonstrate for the first time how faking may be detected on FC measures.

\footnotetext{
${ }^{1}$ Calculated from Tables 5 and 8 in Sun et al. (2021).
} 
The fact that our approach could discriminate accurately between individuals instructed to respond honestly and those instructed to fake on our FC personality measures without our models explicitly incorporating any personality information is perhaps surprising. Many previous methods, including most of those cited above, have attempted to identify faking in terms of response patterns inconsistent with a psychometric model of personality (e.g. using item response theory to determine aberrant responses using person-fit indices; Zickar \& Sliter, 2011). Our approach differs on theoretical grounds, which we argue better captures the faking process. Regardless of a person's trait standing, we assume that faking behavior involves evaluating the relative desirability of the items in a FC block in reference to the goal of presenting better to a hiring committee. Individuals engaging in faking then modify or totally fabricate their responses where necessary to better fit the perceived ideal rank-ordering, given the extent to which the ideal ordering of items is evident. The correlations between our model predictions and self-reported faking behavior suggests that building our approach from this assumption does adequately correspond to the extent of faking behavior, at least as individuals subjectively report engaging in it.

If the goal of an individual engaging in faking is to impress a hiring committee for a particular role, then the ideal response may depend upon the job (Dunlop et al., 2012; Pauls $\&$ Crost, 2005). Contextualizing our detection approach to use job-specific answer keys would thus appear to have both theoretical and applied predictive benefits. The difference in mean faking scores between honest and faking instructions for Study 1 was certainly larger when using our job-specific answer keys (i.e., for the lawyer and kindergarten teacher roles), compared to the generic answer key (for any given job applicant). Likewise, our balanced accuracy and specificity did appear somewhat better in Study 1 for the models using faking scores from job-specific answer keys. Nonetheless, the AUC and sensitivity of the generic model was extremely close to both job-specific models, indicating the benefit of job- 
specificity when applying our proposed methodology remains somewhat ambiguous. Future research could further investigate the utility of job-specificity in our method, and whether certain jobs or job families are similar enough to allow using the same answer key with minimal loss in predictive accuracy.

Our predictive accuracy was substantially higher in Study 1, using a FC measure comprising 48 triplets, compared to Study 2, which used two shorter 20-triplet measures. We suspect that simply having less information with which to classify participants as honest or faking may have negatively impacted our results. This difference seems important from a research perspective, as six separate FC personality measures developed over the past decade have comprised 20 triplets (Brown \& Maydeu-Olivares, 2011; Fisher et al., 2019; Guenole et al., 2018; P. Lee et al., 2018; Watrin et al., 2019; Wetzel \& Frick, 2020), which appears to have become the default in the literature. Our Study 1 measure had more than double this number, and each additional block on a given measure provides more information with which to classify a respondent as honest or faking. Although established commercial measures often use many more blocks than the measures used in either of our studies (e.g., 104 in the OPQ32i, Bartram, 2007), it is possible that the now common 20 block FC measure format found in the academic literature is too short to achieve the best accuracy our approach offers.

Interestingly, Study 2 indicated that the systematic mismatching of the items on desirability minimally impacted predictive accuracy, with the BFT-mixed model performing about as well as the BFT-matched model. This result was somewhat unexpected, as we assumed that having more blocks with clearly 'ideal' answers in the BFT-mixed measure would benefit the discrimination between honest and faking responses. Upon inspection however, the BFT-matched measure contained around eight blocks where at least one of the GMM $\theta$ parameters was $>1$, and with an average Kendall's Tau correlation of $>0.5$ on the ideal ranking. In other words, numerous blocks in the BFT-matched measure had a good deal 
of consensus regarding the ideal response for a psychology masters' applicant. As such, this measure may not have been sufficiently well-matched to strongly impact the detection of faking, relative to the deliberately mismatched BFT-mixed measure. We speculate that strong item desirability matching within FC blocks may still negatively impact the detection accuracy of our method, particularly with matching contextualized to a particular job, although more research is required to substantiate this.

While our method shows substantial promise in empirically detecting faking on FC measures, some comment is warranted on how the model-predicted probabilities may be used in applied personnel selection settings. Based on these predicted probabilities of faking behavior, a practitioner may choose to follow up an applicants' profile at an interview, retest them, or in some cases remove their responses entirely (Hough, 1998). Although excluding participant responses for apparent faking may not substantially impact criterion validities of personality measures, the choice does impact individuals' job offers, particularly where relatively few are ultimately selected (Mueller-Hanson et al., 2003; Schmitt \& Oswald, 2006). It is therefore vital that any faking detection method is evaluated both in terms of its specificity and sensitivity, particularly where there is no opportunity to follow-up a participant's responses or retesting is costly. In both studies, setting our threshold for faking at a default 0.5 predicted probability tended to maximize specificity more so than sensitivity, suggesting that our approach was better able to correctly classify honest respondents relative to those faking, producing fewer 'false positives' (i.e., incorrectly classified honest respondents) that could be wastefully retested or wrongly excluded. Nonetheless, practitioners may need to consider their tolerance for allowing 'false negatives' (i.e., incorrectly classified fakers) to potentially slip through when defining their classification threshold.

\section{Limitations and Future Research}


There are at least two key limitations to our investigation. First and foremost, both studies employed an instructed faking protocol, wherein participants were asked to either respond truthfully or explicitly told to provide the best impression they could for the particular job. This directed faking methodology is necessary to allow researchers to be confident a particular person is indeed faking (i.e., faking behavior is experimentally controlled), but likely exaggerates the behavior. In high-stakes assessment and selection scenarios, faking may be more subtle and strategic, depending on a person's motivation and attitudes among numerous other individual and contextual factors (Birkeland et al., 2006; Roulin et al., 2016). Our classification approach may well successfully identify such extreme faking behavior in practice, but further research is needed to test whether this methodology is as successful at detecting faking under realistic job application responding scenarios. Repeated measurements under honest instructions and later for an actual competitive job application (e.g., Dunlop et al., 2020) may provide a useful way forward in this aim.

Second, our approach could reasonably benefit from incorporating some trait-level personality information. For example, there may be cases where a person's responses happen to correspond with the ideal on enough FC blocks such that their predicted probability of faking is inflated. Nonetheless, this person's responses are in fact consistent with their latent personality trait standing, inclusive of measurement error. This situation would result in false positives that could plausibly be avoided if our approach were combined with something like a person-fit index (Drasgow et al., 1996; Meijer \& Sijtsma, 2001). These indices quantify the consistency of a person's responses with the imposed personality measurement model. Kim \& Moses (2018) have previously proposed such an index to detect aberrant responses on FC measures, which in their simulation study appeared useful in identifying data influenced by faking. Future research empirically testing their methodology and combining it with ours may 
prove a more robust means of identifying faking in light of a person's personality measurements, while attempting to minimize false positives. 
Table 1. Illustrative probabilities of each possible FC response, given an ideal rank-ordering $\pi_{0}$ of Items A ("I am organised") > B ("I am tidy") > C ("I like music").

\begin{tabular}{c|cc|ccc}
\hline \multirow{2}{*}{$\begin{array}{c}\text { Observed } \\
\text { Rank-order, } \\
\pi\end{array}$} & $\begin{array}{c}\text { Distance from } \\
\text { ideal, } d_{j}\left(\pi, \pi_{0}\right)\end{array}$ & $\begin{array}{r}\text { Probability of observed rank-order, } P(\pi), \text { at varying } \\
\text { levels of consensus, } \theta_{j}\end{array}$ \\
\cline { 2 - 6 } & $j=1$ & $j=2$ & $\begin{array}{c}\theta_{1}=0.1 \\
\theta_{2}=0.05\end{array}$ & $\begin{array}{c}\theta_{1}=0.75, \\
\theta_{2}=1.0\end{array}$ & $\begin{array}{c}\theta_{1}=2.0, \\
\theta_{2}=1.25\end{array}$ \\
\hline $\mathrm{A}>\mathrm{B}>\mathrm{C}$ & 0 & 0 & 0.18 & 0.43 & 0.67 \\
$\mathrm{~A}>\mathrm{C}>\mathrm{B}$ & 0 & 1 & 0.18 & 0.16 & 0.19 \\
$\mathrm{~B}>\mathrm{A}>\mathrm{C}$ & 1 & 0 & 0.17 & 0.20 & 0.09 \\
$\mathrm{~B}>\mathrm{C}>\mathrm{A}$ & 2 & 0 & 0.16 & 0.07 & 0.03 \\
$\mathrm{C}>\mathrm{A}>\mathrm{B}$ & 1 & 1 & 0.15 & 0.10 & 0.01 \\
$\mathrm{C}>\mathrm{B}>\mathrm{A}$ & 2 & 1 & 0.15 & 0.04 & $<0.01$ \\
\hline
\end{tabular}

Note: The ideal rank-ordering, $\pi_{0}=[A>B>C]$, with consensus parameters, $\theta_{j}$, arbitrarily selected to illustrate GMM probabilities at comparatively lower, moderate, and higher levels of consensus. 
Figure 1. $k$-fold cross-validation approach

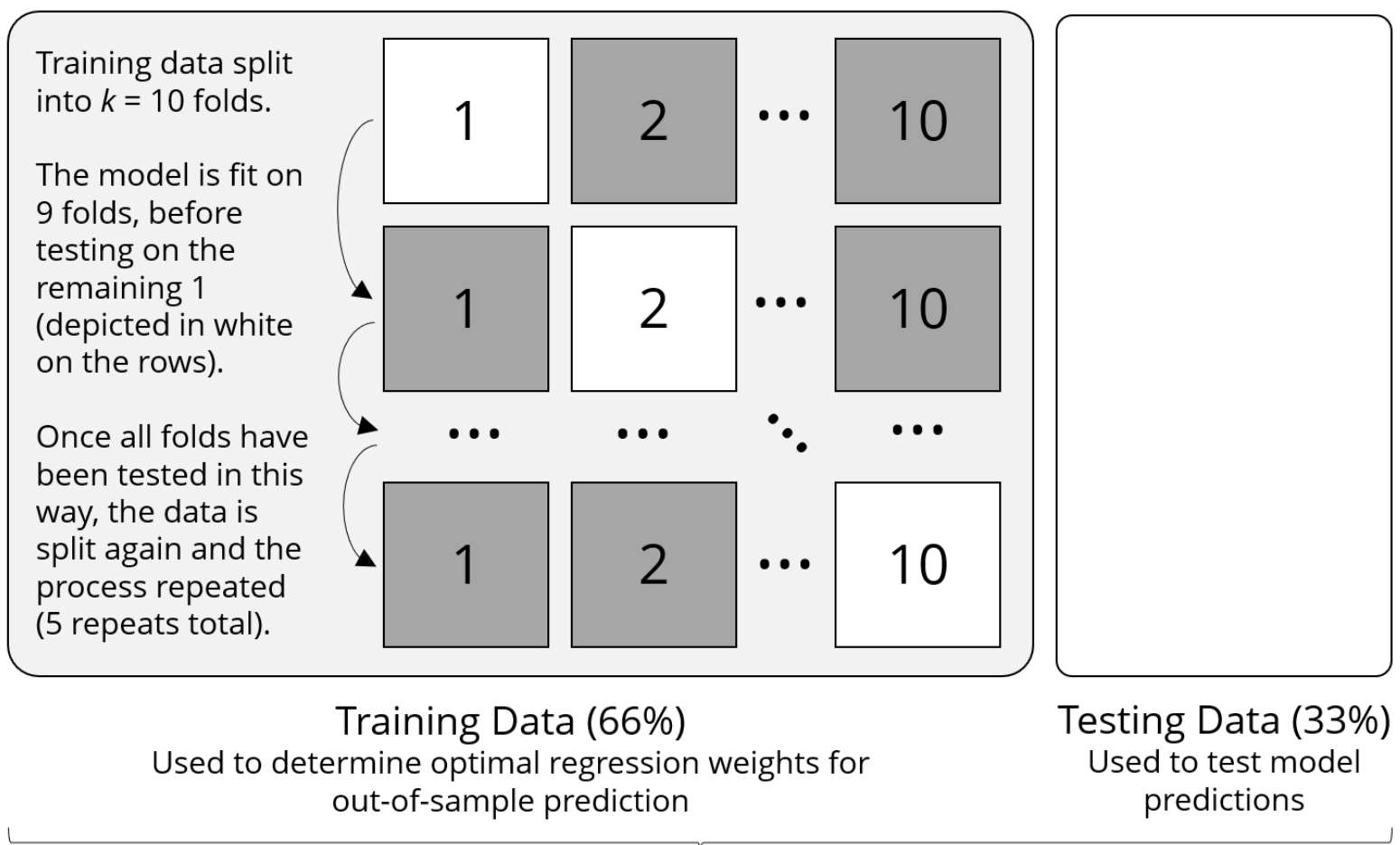

Total Data 
Figure 2. GMM $\theta_{j}$ consensus parameters for all 48 triplets by job applicant condition in Study 1.
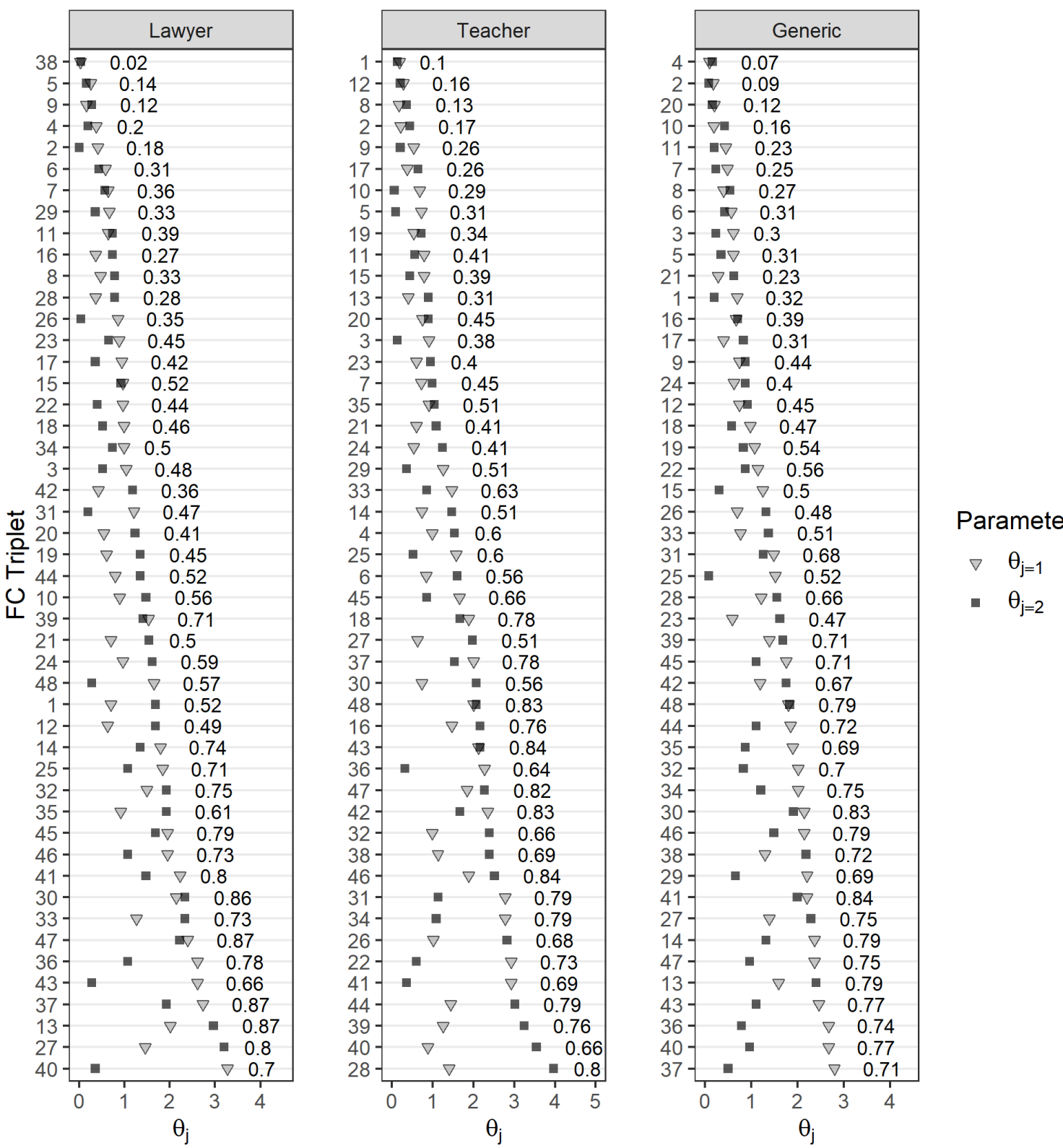

Note: Triplets sorted by largest $\theta_{j}$ value within each block. Numbers represent the average Kendall's $\tau$ correlation between the aggregated ideal rank-ordering, $\pi_{0}$, and respondents' perceived ideal rank-orderings. 
Figure 3. Mean GMM faking scores within individuals by instructions and job condition in Study 1.
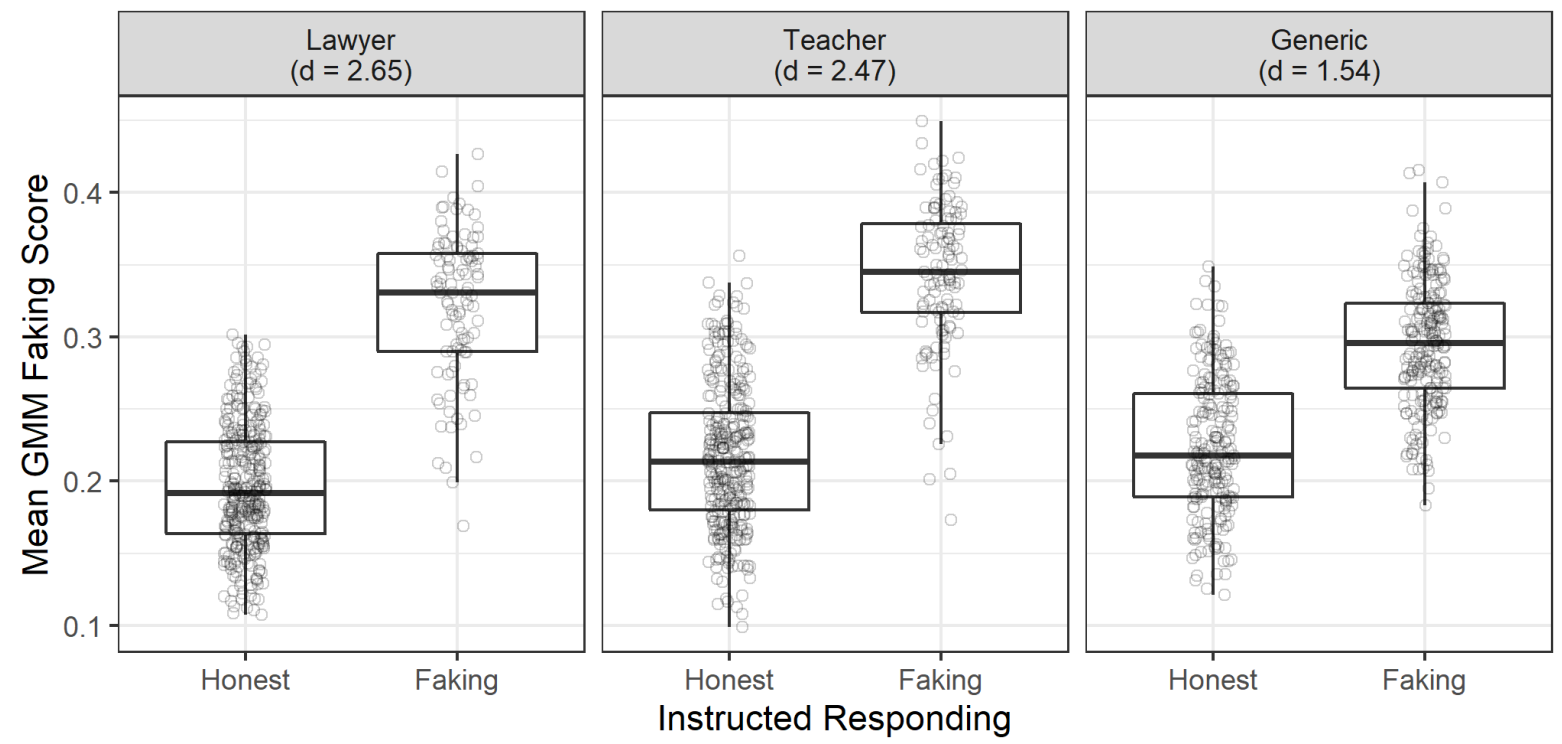

Note: Mean GMM faking scores were calculated by individual across the entire FC measure. Cohen's $d$ mean difference effect sizes between response instructions are presented on the job condition panel. 
Table 2. Predictive performance of elastic net logistic regression (Study 1)

\begin{tabular}{lccccc}
\hline & & \multicolumn{3}{c}{ Predictive Performance (Standard Deviation) } \\
\cline { 3 - 6 } $\begin{array}{c}\text { Cross-validation } \\
\text { sample }\end{array}$ & Model & $\begin{array}{c}\text { Balanced } \\
\text { Accuracy }\end{array}$ & Sensitivity & Specificity & $\begin{array}{c}\text { Area Under } \\
\text { the Curve }\end{array}$ \\
\hline In: Training Data & Lawyer & $.89(.060)$ & $.84(.112)$ & $.93(.052)$ & $.96(.043)$ \\
(Averaged over $k=$ & Teacher & $.90(.050)$ & $.88(.081)$ & $.92(.061)$ & $.97(.028)$ \\
10 folds, 5 repeats) & Generic & $.85(.072)$ & $.86(.089)$ & $.84(.101)$ & $.94(.039)$ \\
Out: Testing Data & Lawyer & .90 & .88 & .92 & .96 \\
$(N=143$ per & Teacher & .92 & .97 & .87 & .97 \\
model) & Generic & .85 & .93 & .77 & .95 \\
\hline
\end{tabular}

Note: Sensitivity represents the model's ability to correctly classify participants engaging in faking, while specificity represents the model's ability to correctly classify honest respondents. Balanced accuracy is quantified as (Sensitivity + Specificity) / 2. The area under the (receiver operating characteristic) curve quantifies model performance across a range of classification thresholds (see Figure 4). 
Figure 4. ROC curves for all three models in Study 1.

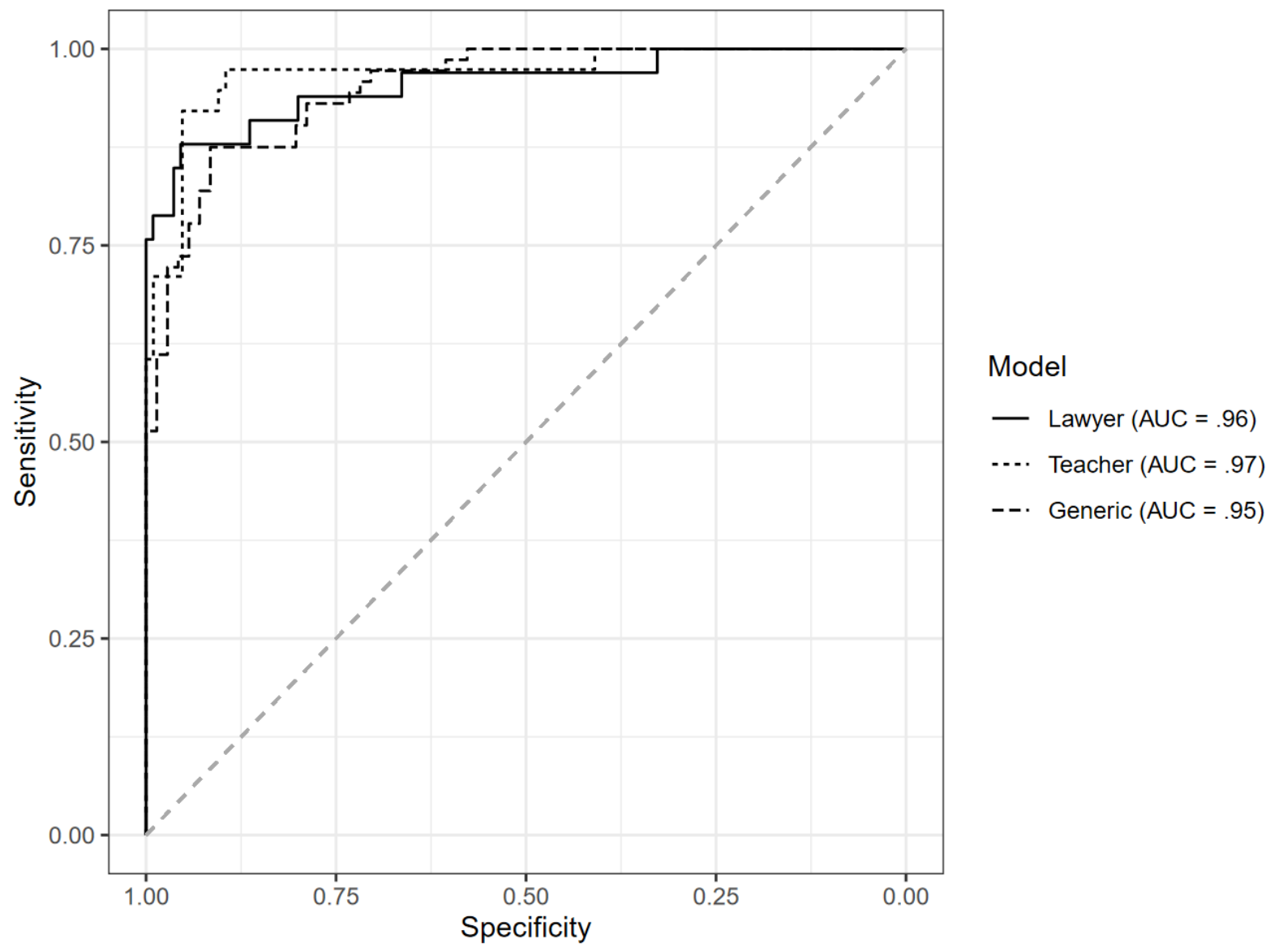

Note: ROC curves show the sensitivity and specificity of models while varying the classification thresholds based on the predicted probability of faking. The area under the curve (AUC) quantifies this, with a value of 1.0 representing perfect sensitivity and specificity. The diagonal line represents a model with no discriminatory ability. 
Figure 5. Predicted probabilities vs. self-reported faking for all models in Study 1.
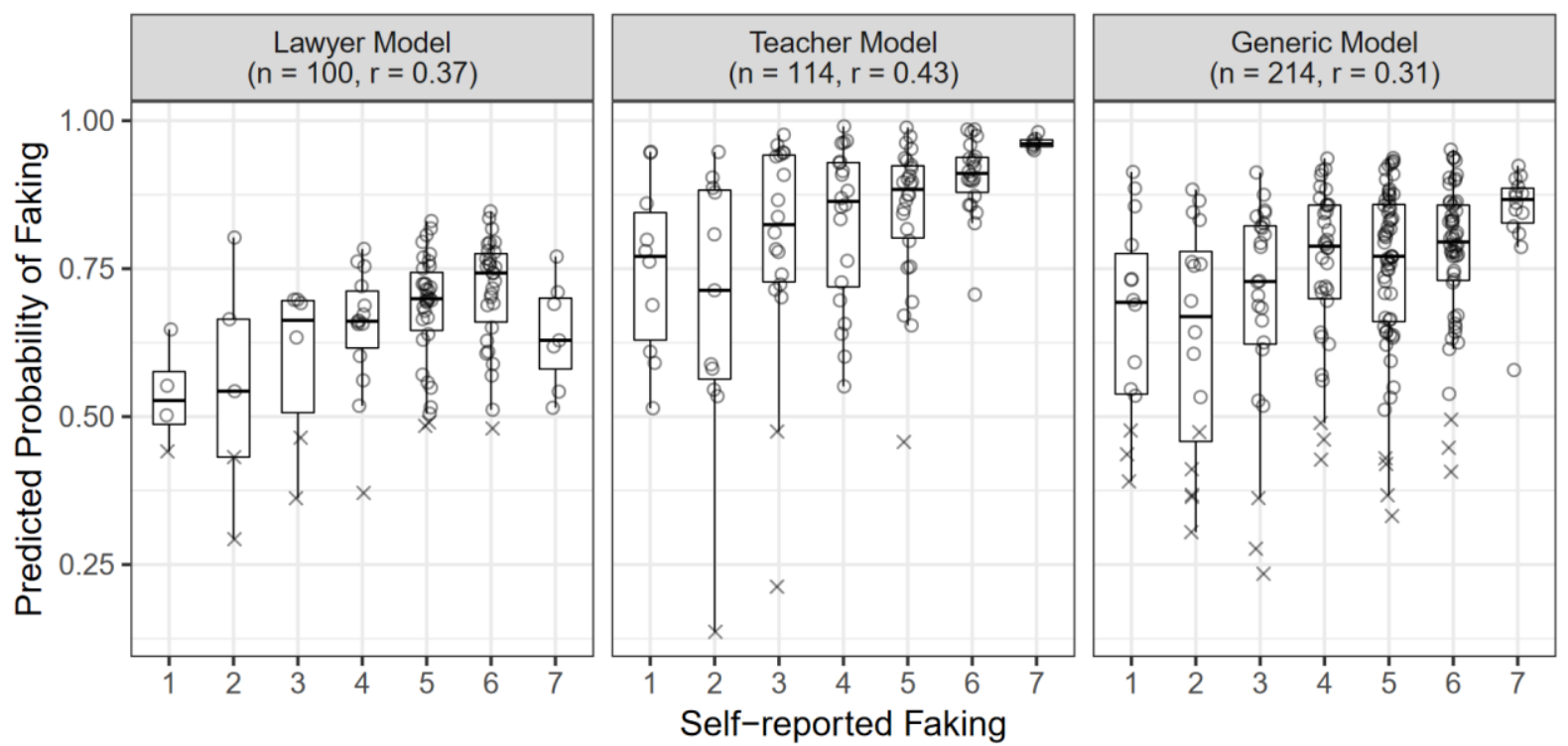

Note: Self-report faking was captured on a single Likert item ranging from 1 ("Not at all my answers were completely honest") to 7 ("Completely - None of my answers were honest"). Points represent correctly classified observations while crosses represent misclassified observations, given a $>0.5$ predicted probability threshold. 
Figure 6. GMM $\theta_{j}$ consensus parameters for all 20 triplets by BFT measure in Study 2
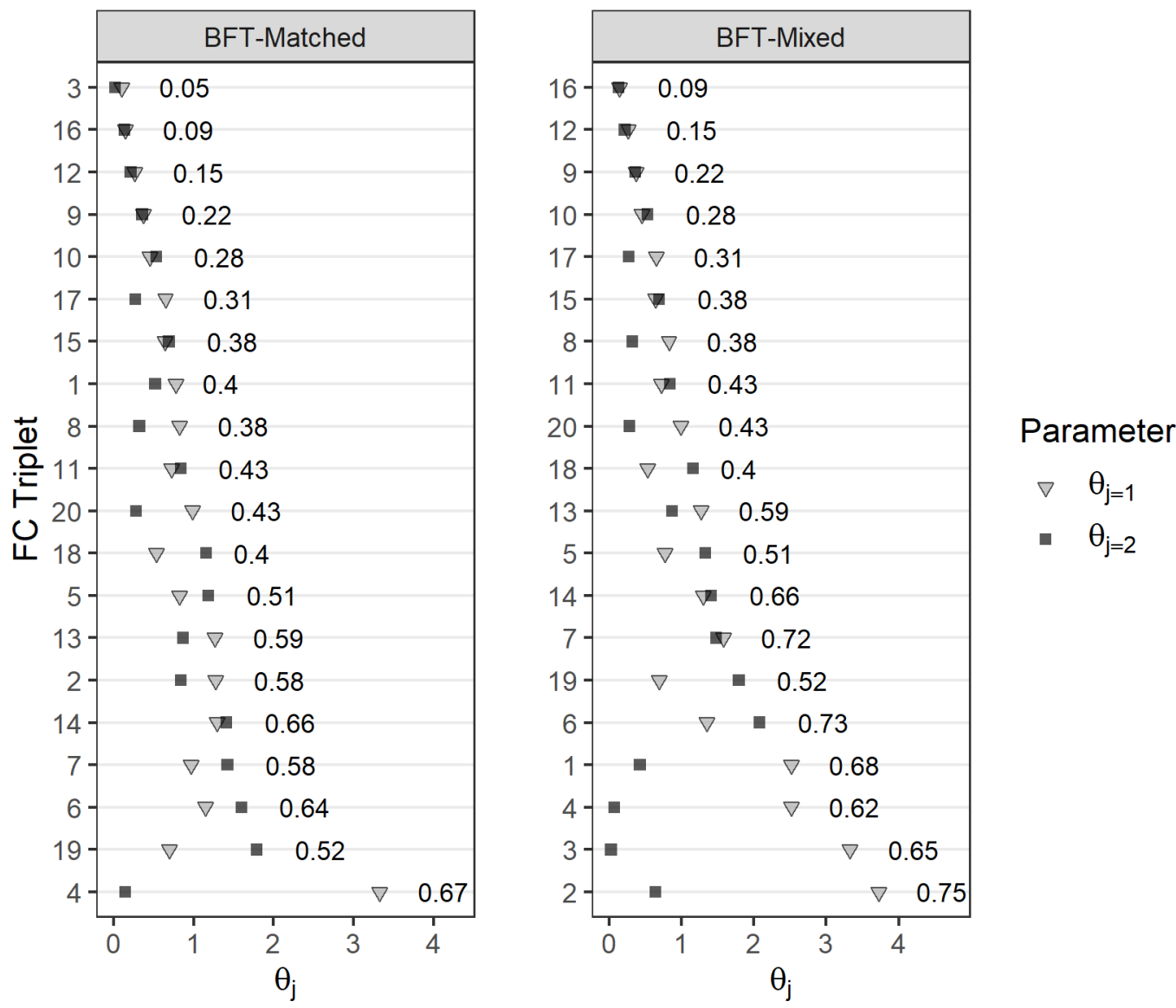

Note: Triplets sorted by largest $\theta_{j}$ value within each block. Numbers represent the average Kendall's Tau correlation between the aggregated ideal rank-ordering, $\pi_{0}$, and respondents' perceived ideal rank-orderings. As the BFT-mixed differed only from the BFT-matched on the first 7 triplets, all results on the remaining triplets (i.e., from 8 onward) are identical between the BFT-mixed and BFT-matched measures. 
Figure 7. Mean GMM faking scores within individuals by instructions and measure type in Study 2

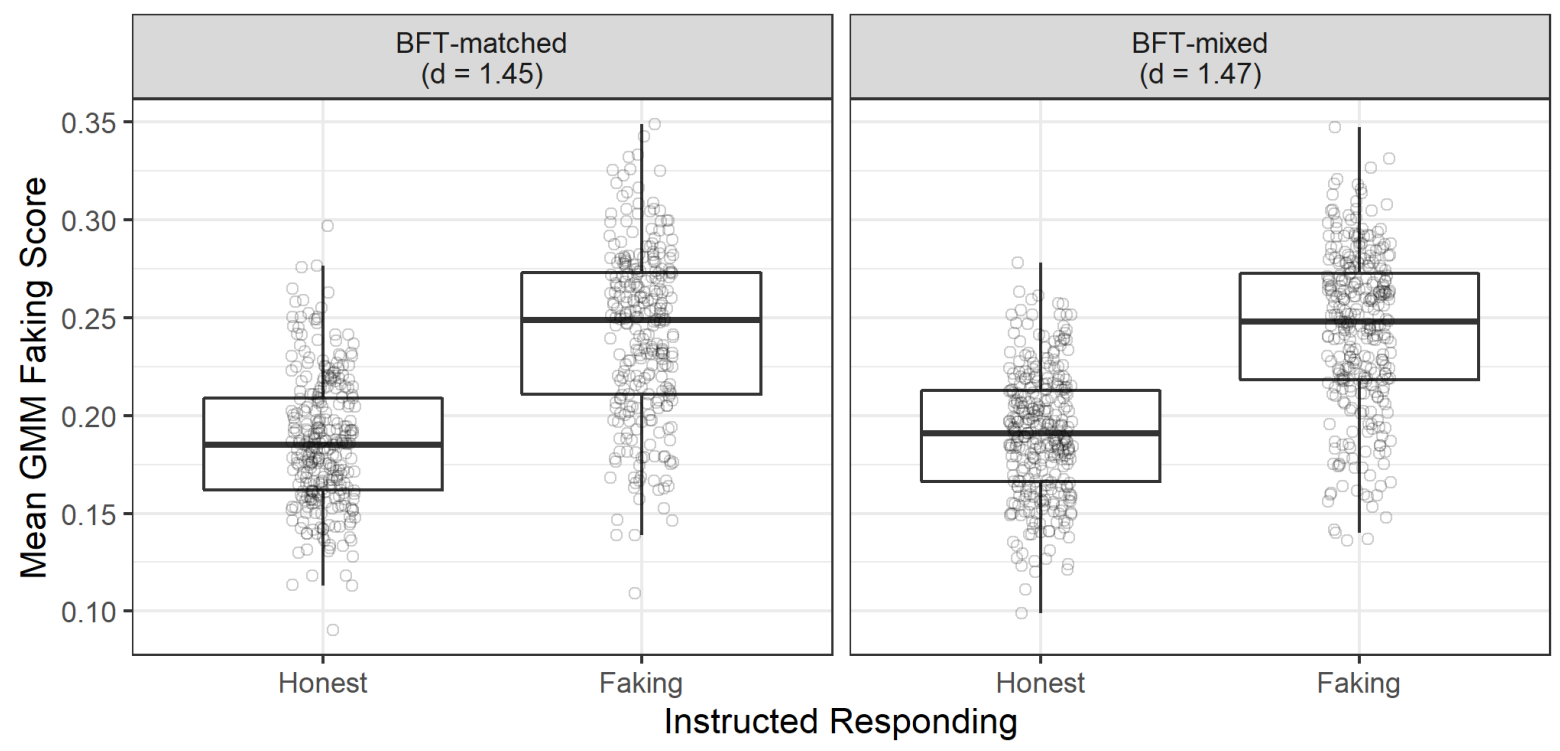

Note: Mean GMM faking scores were calculated by individual across the entire FC measure. Cohen's $d$ mean difference effect sizes between response instructions presented on the measure type panel. 
Table 3. Predictive performance of elastic net logistic regression (Study 2)

\begin{tabular}{lccccc}
\hline & & \multicolumn{3}{c}{ Predictive Performance (Standard Deviation) } \\
\cline { 3 - 6 } $\begin{array}{c}\text { Cross-validation } \\
\text { sample }\end{array}$ & Model & $\begin{array}{c}\text { Balanced } \\
\text { Accuracy }\end{array}$ & Sensitivity & Specificity & $\begin{array}{c}\text { Area Under } \\
\text { the Curve }\end{array}$ \\
\hline $\begin{array}{l}\text { In: Training Data } \\
\text { (Averaged over } k=\end{array}$ & Matched & $.81(.061)$ & $.77(.112)$ & $.86(.077)$ & $.88(.059)$ \\
10 folds, 5 repeats) & Mixed & $.82(.066)$ & $.79(.097)$ & $.86(.082)$ & $.89(.055)$ \\
$\begin{array}{l}\text { Out: Testing Data } \\
(N=192 \text { matched, }\end{array}$ & Matched & .80 & .76 & .84 & .87 \\
$N=212$ mixed) & Mixed & .82 & .78 & .86 & .87 \\
\hline
\end{tabular}


Figure 8. ROC curves for both models in Study 2

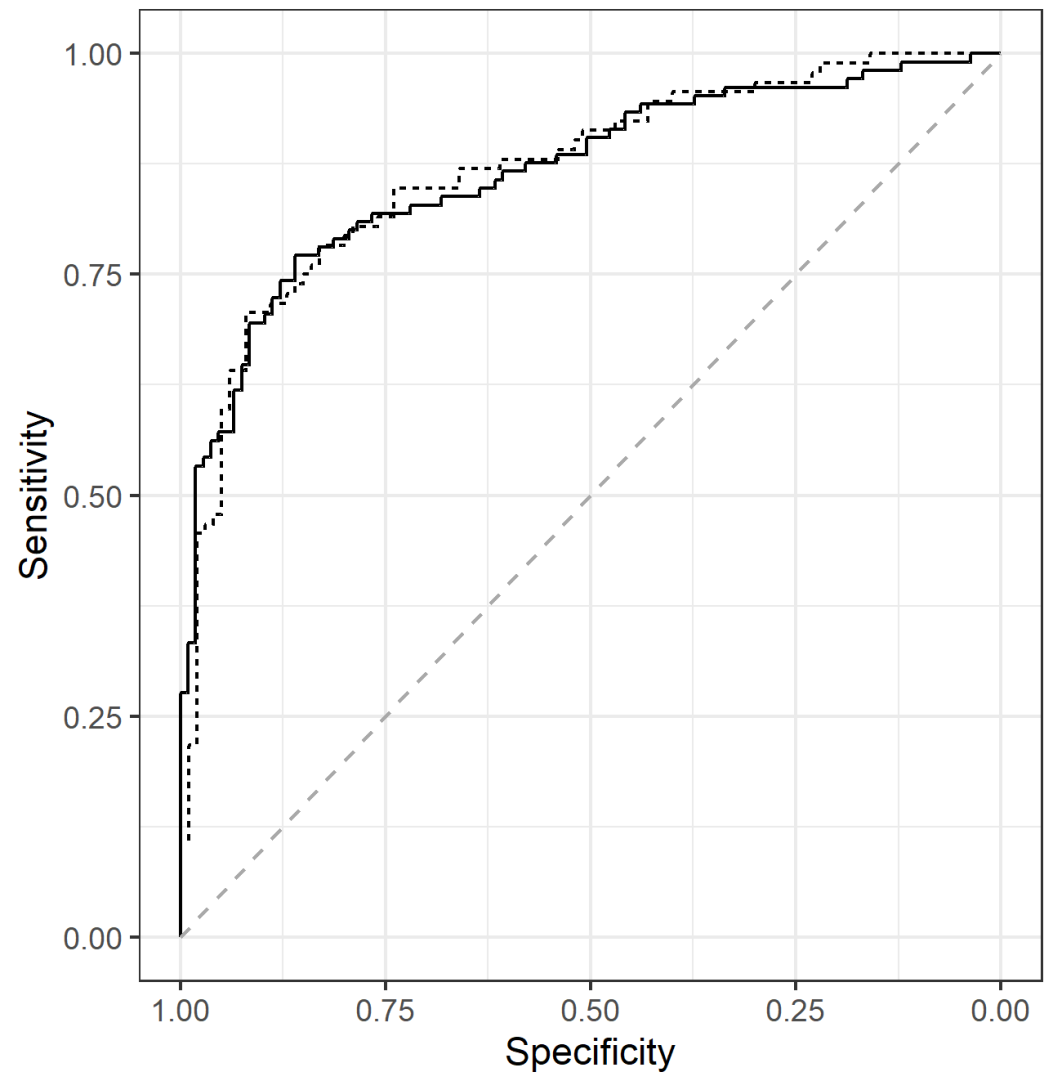

Model

- BFT-mixed $(\mathrm{AUC}=.87)$

-.-- BFT-matched $(\mathrm{AUC}=.87)$ 


\section{References}

Azur, M. J., Stuart, E. A., Frangakis, C., \& Leaf, P. J. (2011). Multiple imputation by chained equations: What is it and how does it work? International Journal of Methods in Psychiatric Research, 20(1), 40-49. https://doi.org/10.1002/mpr.329

Bartram, D. (2007). Increasing validity with forced-choice criterion measurement formats. International Journal of Selection and Assessment, 15(3), 263-272. https://doi.org/10.1111/j.1468-2389.2007.00386.x

Birkeland, S. A., Manson, T. M., Kisamore, J. L., Brannick, M. T., \& Smith, M. A. (2006). A meta-analytic investigation of job applicant faking on personality measures. International Journal of Selection and Assessment, 14(4), 317-335. https://doi.org/10.1111/j.1468-2389.2006.00354.x

Brown, A., \& Maydeu-Olivares, A. (2011). Big Five Questionnaire [Data set]. American Psychological Association. https://doi.org/10.1037/t05430-000

Burns, G., \& Christiansen, N. (2011). Methods of measuring faking behavior. Human Performance, 24, 358-372. https://doi.org/10.1080/08959285.2011.597473

Calanna, P., Lauriola, M., Saggino, A., Tommasi, M., \& Furlan, S. (2020). Using a supervised machine learning algorithm for detecting faking good in a personality selfreport. International Journal of Selection and Assessment, 28(2), 176-185. https://doi.org/10.1111/ijsa.12279

Cao, M., \& Drasgow, F. (2019). Does forcing reduce faking? A meta-analytic review of forced-choice personality measures in high-stakes situations. Journal of Applied Psychology, 104(11). https://doi.org/10.1037/ap10000414

Christiansen, N. D., Robie, C., Burns, G. N., \& Speer, A. B. (2017). Using item-level covariance to detect response distortion on personality measures. Human Performance, 30(2-3), 116-134. https://doi.org/10.1080/08959285.2017.1319366 
Converse, P. D., Pathak, J., Quist, J., Merbedone, M., Gotlib, T., \& Kostic, E. (2010). Statement desirability ratings in forced-choice personality measure development: Implications for reducing score inflation and providing trait-level information. Human Performance, 23(4), 323-342. https://doi.org/10.1080/08959285.2010.501047

Curran, P. G. (2016). Methods for the detection of carelessly invalid responses in survey data. Journal of Experimental Social Psychology, 66, 4-19. https://doi.org/10.1016/j.jesp.2015.07.006

D'Ambrosio, A. (2019). ConsRank: Compute the Median Ranking(s) According to the Kemeny's Axiomatic Approach. https://CRAN.R-project.org/package=ConsRank

Drasgow, F., Levine, M. V., \& Zickar, M. J. (1996). Optimal Identification of Mismeasured Individuals. Applied Measurement in Education, 9(1), 47-64. https://doi.org/10.1207/s15324818ame0901_5

Dunlop, P. D., Bourdage, J. S., de Vries, R. E., McNeill, I. M., Jorritsma, K., Orchard, M., Austen, T., Baines, T., \& Choe, W.-K. (2020). Liar! Liar! (when stakes are higher): Understanding how the overclaiming technique can be used to measure faking in personnel selection. Journal of Applied Psychology, 105(8), 784-799. https://doi.org/10.1037/ap10000463

Dunlop, P. D., Telford, A. D., \& Morrison, D. L. (2012). Not too little, but not too much: The perceived desirability of responses to personality items. Journal of Research in Personality, 46(1), 8-18. https://doi.org/10.1016/j.jrp.2011.10.004

Ferrando, P. J., \& Anguiano-Carrasco, C. (2013). A Structural Model-Based Optimal PersonFit Procedure for Identifying Faking. Educational and Psychological Measurement, 73(2), 173-190. https://doi.org/10.1177/0013164412460049

Fisher, P., Robie, C., Christiansen, N., Speer, A., \& Schneider, L. (2019). Criterion-related Validity of Forced-Choice Personality Measures: A Cautionary Note Regarding 
Thurstonian IRT versus Classical Test Theory Scoring. Personnel Assessment and Decisions, 5(1). https://doi.org/10.25035/pad.2019.01.003

Fligner, M. A., \& Verducci, J. S. (1986). Distance based ranking models. Journal of the Royal Statistical Society: Series B (Methodological), 48(3), 359-369.

Friedman, J., Hastie, T., \& Tibshirani, R. (2010). Regularization paths for generalized linear models via coordinate descent. Journal of Statistical Software, 33(1), 1.

Griffith, R. L., Chmielowski, T., \& Yoshita, Y. (2007). Do applicants fake? An examination of the frequency of applicant faking behavior. Personnel Review, 36(3), 341-355. https://doi.org/10.1108/00483480710731310

Guenole, N., Brown, A. A., \& Cooper, A. J. (2018). Forced-Choice Assessment of WorkRelated Maladaptive Personality Traits: Preliminary Evidence From an Application of Thurstonian Item Response Modeling. Assessment, 25(4), 513-526. https://doi.org/10.1177/1073191116641181

Holden, R. R., \& Book, A. S. (2009). Using hybrid Rasch-latent class modeling to improve the detection of fakers on a personality inventory. Personality and Individual Differences, 47(3), 185-190. https://doi.org/10.1016/j.paid.2009.02.024

Hough, L. M. (1998). Effects of Intentional Distortion in Personality Measurement and evaluation of Suggested Palliatives. Human Performance, 11(2-3), 209-244. https://doi.org/10.1080/08959285.1998.9668032

Hughes, A. W., Dunlop, P. D., Holtrop, D., \& Wee, S. (2021a). Inconsistent with the 'Ideal': Item-level Desirability Influences Faking on Forced Choice Personality Measures [Manuscript submitted for publication].

Hughes, A. W., Dunlop, P. D., Holtrop, D., \& Wee, S. (2021b). Spotting the "Ideal" Personality Response: Effects of Item Matching in Forced Choice Measures for 
Personnel Selection. Journal of Personnel Psychology, 20(1), 17-26.

https://doi.org/10.1027/1866-5888/a000267

Irurozki, E., Calvo, B., \& Lozano, J. A. (2016). PerMallows: An R Package for Mallows and Generalized Mallows Models. Journal of Statistical Software, 71(1), 1-30. https://doi.org/10.18637/jss.v071.i12

James, G., Witten, D., Hastie, T., \& Tibshirani, R. (2013). An Introduction to Statistical Learning (Vol. 103). Springer New York. https://doi.org/10.1007/978-1-4614-7138-7

Kendall, M. G., \& Gibbons, J. D. (1990). Rank correlation methods (5th ed.). Hodder Arnold. Kiefer, C., \& Benit, N. (2016). What is Applicant Faking Behavior? A Review on the Current State of Theory and Modeling Techniques. Journal of European Psychology Students, 7(1), 9-19. https://doi.org/10.5334/jeps.345

Kim, S., \& Moses, T. (2018). The Impact of Aberrant Responses and Detection in ForcedChoice Noncognitive Assessment. ETS Research Report Series, 2018(1), 1-15. https://doi.org/10.1002/ets2.12222

König, C. J., Jansen, A., \& Mathieu, P. L. (2017). What if applicants knew how personality tests are scored? A minimal intervention study. Journal of Personnel Psychology, 16(4), 206-210. https://doi.org/10.1027/1866-5888/a000183

König, C. J., Merz, A. S., \& Trauffer, N. (2012). What is in Applicants' Minds When They Fill Out a Personality Test? Insights from a qualitative study. International Journal of Selection and Assessment, 20(4), 442-452. https://doi.org/10.1111/ijsa.12007

Kuhn, M. (2020). caret: Classification and Regression Training. https://CRAN.Rproject.org/package $=$ caret

Kuhn, M., \& Johnson, K. (2013). Applied Predictive Modeling. Springer-Verlag.

Kuncel, N. R., \& Borneman, M. J. (2007). Toward a new method of detecting deliberately faked personality tests: The use of idiosyncratic item responses. International Journal 
of Selection and Assessment, 15(2), 220-231. https://doi.org/10.1111/j.14682389.2007.00383.x

Lee, M. D., Steyvers, M., \& Miller, B. (2014). A Cognitive Model for Aggregating People's Rankings. PLOS ONE, 9(5). https://doi.org/10.1371/journal.pone.0096431

Lee, P., Lee, S., \& Stark, S. (2018). Examining validity evidence for multidimensional forced choice measures with different scoring approaches. Personality and Individual Differences, 123(July), 229-235. https://doi.org/10.1016/j.paid.2017.11.031

Mandhani, B., \& Meila, M. (2009). Tractable search for learning exponential models of rankings. Artificial Intelligence and Statistics, 392-399.

Meijer, R. R., \& Sijtsma, K. (2001). Methodology Review: Evaluating Person Fit. Applied Psychological Measurement, 25(2), 107-135. https://doi.org/10.1177/01466210122031957

Morgeson, F. P., Campion, M. A., Dipboye, R. L., Hollenbeck, J. R., Murphy, K., \& Schmitt, N. (2007). Reconsidering the Use of Personality Tests in Personnel Selection Contexts. Personnel Psychology, 60(3), 683-729. https://doi.org/10.1111/j.17446570.2007.00089.x

Mueller-Hanson, R., Heggestad, E. D., \& Thornton, G. C. (2003). Faking and selection: Considering the use of personality from select-in and select-out perspectives. Journal of Applied Psychology, 88(2), 348-355. https://doi.org/10.1037/0021-9010.88.2.348

Ng, V., Lee, P., Ho, M.-H. R., Kuykendall, L., Stark, S., \& Tay, L. (2020). The Development and Validation of a Multidimensional Forced-Choice Format Character Measure: Testing the Thurstonian IRT Approach. Journal of Personality Assessment, 1-14. https://doi.org/10.1080/00223891.2020.1739056 
Pauls, C. A., \& Crost, N. W. (2005). Effects of different instructional sets on the construct validity of the NEO-PI-R. Personality and Individual Differences, 39(2), 297-308. https://doi.org/10.1016/j.paid.2005.01.003

Pavlov, G., Shi, D., Maydeu-Olivares, A., \& Fairchild, A. (2021). Item desirability matching in forced-choice test construction. Personality and Individual Differences, 183, 111114. https://doi.org/10.1016/j.paid.2021.111114

Rees, C. J., \& Metcalfe, B. (2003). The faking of personality questionnaire results: Who's kidding whom? Journal of Managerial Psychology, 18(2), 156-165. https://doi.org/10.1108/02683940310465045

Robie, C., Brown, D. J., \& Beaty, J. C. (2007). Do People Fake on Personality Inventories? A Verbal Protocol Analysis. Journal of Business and Psychology, 21, 489-509. https://doi.org/10.2307/25092975

Robie, C., Tuzinski, K. A., \& Bly, P. R. (2006). A survey of assessor beliefs and practices related to faking. Journal of Managerial Psychology, 21(7), 669-681. https://doi.org/10.1108/02683940610690204

Robin, X., Turck, N., Hainard, A., Tiberti, N., Lisacek, F., Sanchez, J.-C., \& Müller, M. (2011). PROC: an open-source package for R and S+ to analyze and compare ROC curves. BMC Bioinformatics, 12, 77.

Roulin, N., Krings, F., \& Binggeli, S. (2016). A dynamic model of applicant faking. Organizational Psychology Review, 6(2), 145-170. https://doi.org/10.1177/2041386615580875

Sackett, P. R., \& Walmsley, P. T. (2014). Which personality attributes are most important in the workplace? Perspectives on Psychological Science, 9(5), 538-551. https://doi.org/10.1177/1745691614543972 
Schmitt, N., \& Oswald, F. (2006). The Impact of Corrections for Faking on the Validity of Noncognitive Measures in Selection Settings. The Journal of Applied Psychology, 91, 613-621. https://doi.org/10.1037/0021-9010.91.3.613

Sun, T., Zhang, B., Cao, M., \& Drasgow, F. (2021). Faking Detection Improved: Adopting a Likert Item Response Process Tree Model. Organizational Research Methods, 10944281211002904. https://doi.org/10.1177/10944281211002904

Watrin, L., Geiger, M., Spengler, M., \& Wilhelm, O. (2019). Forced-choice versus Likert responses on an occupational big five questionnaire. Journal of Individual Differences, 40(3), 134-148. https://doi.org/10.1027/1614-0001/a000285

Wetzel, E., Böhnke, J. R., \& Brown, A. (2016). Response biases. In F. Leong, D. Bartram, F. M. Cheung, K. F. Geisinger, \& D. Iliescu (Eds.), The ITC International Handbook of Testing and Assessment (pp. 349-363). Oxford University Press. http://kar.kent.ac.uk/49093/

Wetzel, E., \& Frick, S. (2020). Comparing the validity of trait estimates from the multidimensional forced-choice format and the rating scale format. Psychological Assessment, 32(3), 239-253. https://doi.org/10.1037/pas0000781

Wetzel, E., Frick, S., \& Brown, A. (2020). Does multidimensional forced-choice prevent faking? Comparing the susceptibility of the multidimensional forced-choice format and the rating scale format to faking. Psychological Assessment, 33(2), 156-170. https://doi.org/10.1037/pas0000971

Wetzel, E., Frick, S., \& Greiff, S. (2020). The Multidimensional Forced-Choice Format as an Alternative for Rating Scales. European Journal of Psychological Assessment, 36(4), 511-515. https://doi.org/10.1027/1015-5759/a000609

Zavala, A. (1965). Development of the forced-choice rating scale technique. Psychological Bulletin, 63(2), 117-124. 
Zickar, M., \& Sliter, K. (2011). Searching for Unicorns: Item Response Theory-Based Solutions to the Faking Problem. New Perspectives on Faking in Personality Assessment. https://doi.org/10.1093/acprof:oso/9780195387476.003.0038

Ziegler, M., MacCann, C., \& Roberts, R. D. (2012). Faking: Knowns, unknowns, and points of contention. In M. Ziegler, C. MacCann, \& R. D. Roberts (Eds.), New perspectives on faking in personality assessment. (pp. 3-16). Oxford University Press.

Zou, H., \& Hastie, T. (2005). Regularization and variable selection via the elastic net.

Journal of the Royal Statistical Society: Series B (Statistical Methodology), 67(2), $301-320$. 